\begin{tabular}{|c|l|}
\hline Title & Multi-functions of hydrogel with bilayer-based lamellar structure \\
\hline Author(s) & Haque, Md. A namul; Gong, Jian Ping \\
\hline Citation & $\begin{array}{l}\text { Reactive \& Functional Polymers, 73(7), 929-935 } \\
\text { https://doi.org/10.1016/.reactfunctpolym.2013.01.016 }\end{array}$ \\
\hline Issue Date & 2013_07 \\
\hline Doc URL & http://hdl.handle.net/2115/53083 \\
\hline Type & article(author version) \\
\hline File Information & Haque_Manuscript_Reactive and Functional Polymer.pdf \\
\hline
\end{tabular}

Instructions for use 


\section{Multi-functions of Hydrogel with Bilayer-based Lamellar Structure}

\section{Md. Anamul Haque ${ }^{\mathrm{a}}$ and Jian Ping Gong ${ }^{\mathrm{b}} *$}

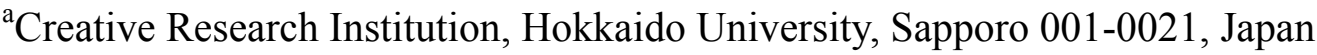

${ }^{\mathrm{b}}$ Faculty of Advanced Life Science, Hokkaido University, Sapporo 060-0810, Japan

*Tel. \& Fax: +81-11-706-2774, E-mail: gong@sci.hokudai.ac.jp

*Corresponding Author

Key Words: Self-assembly, Multilayer diffraction, Tunable structure color, Anisotropy, Stratified layer. 


\begin{abstract}
A novel hybrid hydrogel has been developed by combining bilayer-based lamellar structure of a self-assembled polymer surfactant and polymer network of conventional hydrogel system. A wide range of lamellar structure from micro-domain up to macro-domain (cm-scale) has been successfully generated in the hydrogel. Flat, infinitely large, and perfectly aligned lamellar macro-domain was formed by applying mechanical shear to the gel forming precursor solution containing monomer, cross-linker, and initiator. The obtained hydrogel system contains macroscopic, single-domain, periodical stacking of integrated microscopic lamellar bilayers inside the polymer matrix of the hydrogel. Periodical stacking of the bilayers in the hydrogel selectively diffract visible light to exhibit magnificent structural color. Due to the uniaxial orientation of the bilayer, the hydrogel possesses superb functions that have never been realized before, such as the one-dimensional swelling, anisotropic Young's modulus, anisotropic molecular permeation, and diffusion. Furthermore, the hydrogel exhibits excellent color tuning ability over a wide spectrum range by mechanical stimuli.
\end{abstract}




\section{Introduction}

Hydrogels are soft and wet materials usually composed of three dimensional polymer network structure and a large amount of water interposed inside the network structure. They have become extensively attractive due to their ability to simulate biological tissues and respond reversibly to the external stimuli [1-10]. However, most conventional soft and wet hydrogels usually show extremely poor functions due to their amorphous structure, i.e., random cross-linking polymer chain at molecular level, in contrast with the natural bio-tissue that possesses well-defined hierarchy structure from molecular level to macroscopic scale. The well ordered anisotropic structures of the biological tissues enable highly elaborate functions of living organisms [11-15]. For example, actins and myosin show a liquid crystalline anisotropic structure in a muscle sarcomere, which contributes the smooth motion of muscle fibers and muscle contraction in a specific direction [16-18].

Although several hydrogels with high strength and toughness have been developed in last decade, they also possess amorphous structure in contrast to the bio-tissue [19-23]. Therefore, it is a challenging task for the scientists to introduce well-define structure in hydrogel which serves highly elaborate functions. Recently, a method has been fabricated to introduce well-define lamellar bilayer structure of self-assembled surfactant inside polymer hydrogel. In the past decades, Tsujii et al. have discovered a micro-scale lamellar structure of a self-assembled surfactant in the aqueous solution which can be fixed in the network structure of hydrogel by polymerization in mixed monomer solution. However, this micro-domain lamellar superstructure in hydrogel plays negligible improvement in the functional hybrid gel material due to the poly-domains of the lamellar phase in macro-scale [24-27]. Very recently, Gong's group has developed a successful method for formation of macroscopic (integrated microscopic) single-domain lamellar bilayer structure with periodical stacking inside the polymer matrix of the hydrogel $[28,29]$. The hybrid hydrogel system 
already attracted great attention in material science because two novel phenomena, excellent mechanical performances and tunable structural color, are emerged in one material.

In this article, the authors intend to discuss the self-assembly behavior of a polymeric surfactant in water as well as in polymer network of hydrogel. The polymeric surfactant usually undergoes self-assembly and forms micro-scale lamellar domains with certain periodic spacing in aqueous solution showing iridescence and while introduced in the hydrogel, the rigid lamellae also diffract visible light to exhibit magnificent structural color in the hygrogel. Periodically ordered uni-axial bilayers in the hydrogel not only diffract visible light but also serve as reversible sacrificial bonds providing excellent mechanical performance of the hydrogel such as strength, shape-recovery. Furthermore, stratified soft and hard layer structure allow the hydrogel to exhibit superb anisotropic property such as one dimensional swelling, anisotropic modulus and excellent color tuning ability under mechanical stimuli as like a soft tactile sensor or a mechanical sensor.

\section{Iridescence resulting from poly-domain lamellae of self-assembled surfactant}

Pure dodecyl glyceryl itaconate (DGI) forms lamellar liquid crystalline droplets in water at $55^{\circ} \mathrm{C}$ with the diameter of several micrometers $(\sim 10 \mu \mathrm{m})$ [25]. The spacing distance between bilayer membranes in these liquid crystals is in several nanometers $(\sim 6 \mathrm{~nm})$. With the presence of small amounts of ionic surfactants, the bilayers are separated apart and forms lamellar domains in solution with certain periodic spacing in the scale of $50-350 \mathrm{~nm}$. The lamellar domains are periodically ordered in micro-scale but randomly oriented in the bulk solution from macroscopic view (Figure 1a). In the DGI systems, it is found that ionic surfactants are necessary to change from the lamellar liquid crystalline droplets of pure DGI having the spacing distance of several $\mathrm{nm}$ to the iridescent lamellar phases with a periodic spacing of several $100 \mathrm{~nm}$. For the pure DGI samples without any ionic surfactants, the liquid crystalline droplets are kept unchanged even after being left in water bath at a higher 
temperature than the Kraff point for a week. The electrostatic repulsive force of the head group of the ionic surfactant separates apart the DGI bilayers at the initial moment, and DGI lamellar liquid crystalline droplets undergo the transition to swollen lamellar bilayer membranes. The swollen bilayer systems thus obtained show the iridescent colors in the visible wavelength region depending on the SDS concentration. One can find that the iridescent color shifts to blue side with increasing concentration of SDS. Figure 1b shows the color photographs of selected iridescent DGI solutions as a function of SDS concentration. When the concentration of DGI is fixed to $1.63 \mathrm{wt} \%$ and the molar ratio of SDS is increased from 1:4000 to $1: 40$ (from $1.09 \times 10^{-5}$ to $1.09 \times 10^{-3} \mathrm{molL}^{-1}$ ), the color of the iridescent solution changes from pink to deep blue. The freeze-fracture TEM images of DGI solutions in which the ratios of SDS to DGI are 1:4000 and 1:40, respectively are also shown in Figure 1b. When the molar ratio of SDS to DGI is 1:4000, the lamellar sheets can be clearly seen in the TEM image. The bilayers occupy the whole space of the solution. However, when the molar ratio of SDS is increased to 1:40, the onion-like and/or myeline-like lamellae are found [25].

According to the packing theory [30], the shape of the surfactant aggregates is mainly governed by the packing parameter of $v / L s$, where $v, L$ and $s$ are the volume, the length of the alkyl chain and the effective surface area of the head group of surfactant molecule, respectively. DGI molecule may be in truncated conical shape and tends to form flat lamellae, while the molecule of SDS is wedge-shaped and favors to form spherical micelles. When the ratio of SDS is increased, it is reasonable that the structure of DGI bilayers will change from flat lamellar sheets to lamellar vesicles consisting of curved bilayer membranes. Once the separated onion-like vesicles are formed, the bilayer membranes cannot occupy the whole space of the solution, resulting in the emergence of some vacant spaces. The interplanar distance between bilayer membranes decreases in the above situation since the space for the bilayer membranes becomes smaller. It is well evident that DGI forms flat 
lamellar sheets at low concentration of SDS (1:4000 relative to DGI) which is mainly focused hereafter.

The periodic micro-domain lamellar structure of DGI is maintained even in the aqueous solution of acrylamide monomer (AAm), $N, N$-methylene bis(acrylamide) (MBAA), and initiator. The iridescent solution of DGI was polymerized by UV light and the lamellar structure of the polymeric DGI became immobilized inside the network structure of polyacrylamide (PAAm) gel. The obtained hybrid gel (PDGI/PAAm) exhibits iridescent multi-colors, and is isotropic in macroscopic scale, similar to the precursor solution in which lamellar bilayer domains are randomly oriented [27]. Therefore, the bulk hydrogel with microscopic lamellar domains showed poor anisotropy in the optical, swelling, and modulus and weak mechanical strength.

\section{Formation of single-domain lamellae in macroscopic gel}

Tsujii et al developed the hybrid hydrogel that contains microscopically anisotropic bilayer structure exhibits iridescent color because the bilayer micro-domains distributed randomly in bulk gel from macroscopic point of view [27]. Haque et al succeeded to fabricate the structure uniformity of the hybrid gel by aligning the bilayer sheets in one direction before polymerization, i.e, macroscopic uni-domain lamellar bilayer structure fixed inside the polymer matrix of the PAAm hydrogel after polymerization [28] which is summarized in

Figure 2. By applying a strong shear to the precursor solution that contains micro-domain bilayers, several thousand of the lamellar bilayers are aligned uni-axially along the substrate surface up to the $\mathrm{cm}$ scale or macroscopic scale. Aqueous solution containing prescribed amounts of DGI, $0.025 \mathrm{~mol} \%$ SDS (1:4000 relative to DGI), $2 \mathrm{M}$ AAm, $0.05-0.1 \mathrm{~mol} \%$ MBAA, and $0.1 \mathrm{~mol} \%$ Irgacure 2959 (the molar percentages are relative to AAm) was left to stand in a water bath at $55^{\circ} \mathrm{C}$ for about $4 \mathrm{~h}$ until the formation of stable lamellar bilayers. The solution was purged in an argon atmosphere to remove the dissolved oxygen. The precursor 
solution was injected, with a pipette, into the $500-\mu \mathrm{m}$-thick reaction cell of $10 \mathrm{~cm}$ length and $1 \mathrm{~cm}$ width. The injection speed of the solution was about $5 \mathrm{cms}^{-1}$, corresponding to a shear rate of $200 \mathrm{~s}^{-1}$. As the precursor solution contained randomly oriented micro-domains of stable lamellar bilayers, the strong shear induced the bilayer micro-domains to align in one direction parallel to the glass substrates in the cm-scale. By UV polymerization for $8 \mathrm{~h}$ at $50^{\circ} \mathrm{C}$ under an argon gas atmosphere, the uni-axial lamellar structure of PDGI was immobilized and stabilized inside the PAAm network. Therefore, an anisotropic hydrogel film (thickness: $500 \mu \mathrm{m}$, length: $10 \mathrm{~cm}$, width: $1 \mathrm{~cm}$ ) was obtained in which lamellar bilayer sheets oriented parallel to the wide surface of the film. At the as-prepared state, the sheet-shaped anisotropic PDGI/PAAm gel films are almost transparent and show Bragg's diffraction in ultra-violet wavelength band $\left(\lambda_{\max } \sim 200-400 \mathrm{~nm} ; d \sim 85-170 \mathrm{~nm}\right)$, but after reaching equilibrium swelling in water, they exhibit excellent visible color $\left(\lambda_{\max } \sim 400-700\right.$ nm) with an inter bilayer spacing, $d \sim 170-300 \mathrm{~nm}[28]$.

\section{Anisotropic property and stratified layer structure model}

The photographs of the PDGI/PAAm gels prepared at various DGI concentrations are shown in Figure 3a. The lamellar bilayers in the gel are in parallel to the screen of photographs. The lamellar alignment is also shown in the 3D illustration of the gel (Figure 3b). The very pure color of the gel in cm-scale due to uniform Bragg's diffraction indicated the macroscopic or cm-scale unidirectional orientation or uni-axial pattern of bilayer in the gel. The formation microscopic as well as macroscopic uni-axial bilayer structure of the PDGI/PAAm gel has been clearly observed by the transmission electron microscope (TEM) observation, small angle $\mathrm{x}$-ray scattering (SAXS), and polarizing optical microscope (POM). The POM images shown in Figure 3(c-f) were taken from the cross section of the plate-like gel sample. The image appeared as complete dark under the crossed polarizers at a crossing angle of $0^{\circ}$ (Fig. 3c), whereas at a $45^{\circ}$ crossing angle (Fig. 3d), a perfect bright image was observed. These 
results demonstrate that the lamellar bilayer of DGI was oriented unidirectionally parallel to the top surface of the sheet-like gel. However, the gel prepared without shear flow produce images with similar birefringence level when rotaing by an angle of $45^{\circ}$ (partially bright) indicating the random lamelar orientaion in the gel.

Unlike regular photonic crystals with three-dimensional lattice structures, the lamellar gel contains unidirectional layers and exhibits a one-dimensional optical lattice. The thickness of one PDGI lamellae is $4.7 \mathrm{~nm}$ [24] and the distance between two next neighboring PDGI layers, $d$, is $150-300 \mathrm{~nm}$ depending on the DGI concentration and the swelling degree of PAAm networks. Owing to the global uni-axial orientation of the lamellar bilayers, the swellable PAAm network can swell freely in the direction perpendicular to the bilayers. However, the free swelling of PAAm layer is completely constrained by the rigid, non-swellable PDGI layers (Figure 4a). Therefore, the gel has a strong anisotropy in its properties: it exhibits one-dimensional swelling in the direction perpendicular to the lamellar bilayers while the swelling in the direction of the bilayers is completely constrained. The swelling ratio of the PDGI/PAAm gel along the thickness axis (perpendicular to PDGI bilayers) increased up to 2.2 with the progress of time whereas no swelling is observed at all along the length axis (parallel to bilayers) (Figure $4 \mathbf{b}$ ). This indicates the one-dimensional swelling behavior perpendicular to the bilayers direction, which is in contrast to the single PAAm gel that shows isotropic swelling [31].

Concomitant with the appearance of the anisotropic swelling, the PDGI/PAAm gel showed clear anisotropy in modulus. The overall modulus of the gel parallel to the bilayers direction $(400 \mathrm{kPa})$ is 20 time higher than that perpendicular to the bilayers $(10 \mathrm{kPa})$. Therefore, the individual modulus for a single bilayer and the PAAm layer has been calculated. At room temperature, a single PDGI bilayer has a high modulus in the order of several $\mathrm{MPa}(\sim 4000 \mathrm{kPa})$ and the PAAm matrix is much softer than the bilayer, with a 
modulus of few $\mathrm{kPa}(5 \mathrm{kPa})$. Thus, the PDGI/PAAm hydrogel is said to have a stratified structure consisting of two alternating layer structures, one is rigid PDGI and another is soft PAAm layers, similar to the structure found in some biological systems showing structural colors, such as in iridophore of tropical fishes [33,34] and nacre [34] which is crucially important to be a tough material.

By accounting for both the swelling PAAm layers (SLs) and nonswelling PDGI lamellar layers (NLs), a simple yet effective model was formulated by Hong et al for the anisotropic property of lamellar gels (Figure 5a). The deformation and swelling of the soft SLs made from hydrophilic polymer networks (HPNs) is constrained by the stiff NLs, made from glassy polymer or surfactant bilayers. For simplicity, the principal directions of deformation are assumed to be either parallel or perpendicular to the lamellae. The synthesized state is considered as the reference state in which the HPN in the SLs is stretched isotropically and homogeneously due to the presence of a small amount of solvent during synthesis $[28,36]$. The stretch of the as synthesized HPN with respect to the dry state is denoted as $\lambda_{0}$. The NLs, on the other hand, are assumed to extend freely in this state. In a deformed state, $\lambda_{1}, \lambda_{2}$, and $\lambda_{3}$ are assumed the principal stretches in the SLs with respect to the reference. More specifically, $\lambda_{3}^{\prime}$ be in the through-thickness direction of lamellae. By applying the model, the resulting calculated free-swelling stretches parallel $\left(\lambda_{1}\right)$ and perpendicular $\left(\lambda_{3}\right)$ to the lamellae are plotted in Figure $5 \mathbf{b}$ as functions of the DGI concentration. The results show relatively good agreement with the experimental measurements [28]. At low DGI concentration, the lamellar structure may be less ordered and the deformation may not be affine due to the large distance between neighboring bilayers. The relatively large discrepancy is thus expected. The modulus, namely the initial slope of the stress-strain curve in uniaxial tension and compression, can also be evaluated through linearizing the stress-stretch relation at the small-strain limit. Using the same set of material parameters as in the swelling 
calculation, the tensile modulus in the direction of the bilayers, $E_{1}$, and that in the perpendicular direction, $E_{3}$ was computed. The results are plotted in Figure $\mathbf{5 c}$ and also compared to the available experimental data [28]. The prediction of the model shows good agreement at a DGI concentration higher than $0.06 \mathrm{molL}^{-1}$.

\section{Mechano-responsive color tunability}

PGDI/PAAm gel developed by author's group exhibits magnificent structural color as a result of multilayer Bragg's diffraction of visible light produced by the periodic multi-lamellar planes. The color of the gel can interestingly be tuned reversibly by applying compressive mechanical stress. When the gel was compressed perpendicular to the lamellar layer direction, the gel changed its color under the stress/strain and then returned to its initial color after the removal of the stress/strain. The photographs of the gel at various compression states and their corresponding reflection spectra are shown in Figure 6a. The color as well as the reflection peak shifted from the red to the blue wavelength region with the increase of applied strain. The wavelength at maximum of the spectrum, i.e., peak wavelength $\left(\lambda_{\max }\right)$ decreased from $\sim 600 \mathrm{~nm}$ to $\sim 415 \mathrm{~nm}$ with an increase in the strain, $\varepsilon$, from 0 to 0.64 (Figure 6b). During compression, most of the deformation localized only in the soft PAAm layer which cause a decrease in the lamellar distance whereas the rigid PDGI lamellae might experience slight distortion by lateral expansion accompanied with the compression. The smaller the inter lamellar distance, $d$, results in the Bragg's diffraction of visible light in the lower wavelength region and the corresponding blue shift of gel color.

The PDGI/PAAm gel also exhibits tunable color from orange to blue by applying tensile deformation in direction parallel to the lamellar plane (Figure 7a). This color change is reversible, i.e., the gel changes its color under an applied tensile stress/strain and then returns to its initial color and dimensions after releasing of the stress/strain. The wavelength at maximum of the reflection spectrum $\left(\lambda_{\max }\right)$ has found to be decreased with the increase in 
tensile strain (Figure 7b). Since elongation accompanies with the decrease in gel thickness, i.e., compressive deformation perpendicular to stretching direction, inter lamellar distance, $d$, decreases with elongation (Figure $\mathbf{7 b}$ ). As a result, lower wavelength light is reflected and consequent blue shift of color is observed. This color tuning phenomenon can be repeated for many times and the gel behaves like a rubber during stretching and releasing.

Most polymer materials that exhibit structural color are based on the incorporation of periodically packed colloidal particles into the polymer matrix, or the formation of a porous polymer matrix by etching away the precursor colloidal particles [37-45]. These photonic crystalline materials are therefore isotropic in structure, they have poor color quality and weak mechanical response, and their color tuning ability covers narrow wavelength band $(<70 \mathrm{~nm})$. On the other hand, the lamellar hydrogel sustains large deformation (compressive $\sim 90 \%$ and tensile $\sim 2200 \%)$ and tunes color over the entire visible wavelength region $\left(\Delta \lambda_{\max }=300 \mathrm{~nm}\right)$ under the compressive mechanical deformation. Due to this large structural color tuning, this gel could be potentially used as a stress/strain or mechano-responsive sensor.

\section{Conclusions}

Self-assembly of a polymerizable surfactant-like molecule can form micro-domain lamellar structure in solution which is further incorporated and fixed in conventional polymer network to form hydrogel. In solution stage, the microscopic poly-domain lamellar structure has been integrated to form macroscopic single-domain flat lamellae in presence of gel forming monomer, cross-linker, and initiator. The infinitely large, flat lamellar structure was trapped and immobilized inside gel network by polymerization. This idealized microstructure, in which both swellable, soft polymer network and non-swellable, hard lamellae are flat, infinitely large, and perfectly aligned, generated a perfect anisotropic hydrogel with stratified structure that has not been realized before. The hydrogel exhibits robust functions such as beautiful structural color, one-dimensional swelling, anisotropic modulus, stress induced color 
tuning, and excellent mechanical strength which play important roles in diverse applications. The unique feature of this hydrogel is not only reversible physical association by self-assembly but also the random coil polymer bond itself in the aggregates. The retraction of the extended polymer chain of the deformed bilayers into random coil configuration enhances the ability to self-recover of its initial shape, color, and strength. Therefore, this hydrogel system would guide the scientist to fabricate more strong, tough, and reversible bond in soft material and also to create as a new class of soft tactile sensor or mechanical sensor.

\section{ACKNOWLEDGEMENTS}

This study was supported by a Grant-in-Aid for Specially Promoted Research (No. 18002002) from the Ministry of Education, Science, Sports and Culture of Japan. 


\section{References}

[1] N. A. Peppas, Hydrogels in Medicine and Pharmacy, CRC Press, Boca Raton, FL; 1987.

[2] J. D. DeRossi, K. Kajiwara, Y. Osada, A. Y. Yamauchi, Polymer gels-fundamentals and biomedical applications, Plenum Press, New York; 1991.

[3] N. A. Peppas, R. Langer, Science 263 (1994) 1715.

[4] T. Tanaka, I Nishio, S. T. Sun, S. Ueno-Nishio, Science 218 (1982) 467.

[5] Y. Osada, H. Okuzaki, H. Hori, Nature 355 (1992) 242.

[6] G. Chen, A. S. Hoffman, Nature 373 (1995) 49.

[7] D. J. Beebe, J. S. Moore, J. M. Bauer, Q.Yu, R. H. Liu, C. Devadoss, B. H. Jo, Nature $404(2000) 588$.

[8] J. P. Gong, Y. Osada. Adv Mater 10 (1998) 827.

[9] J. A. Rowley, G. Madlambayan, D. J. Mooney, Biomaterials 20 (1999) 45.

[10] J. Chen, H. Park, K. Park, J. Biomed. Mater Res 44 (1999) 53.

[11] Y. M. Evdokimov, T. V. Nasedkina, V. I. Salyanov, N. S. Badaev, Mol. Biol. 30 (1996) 219.

[12] M. Duvert, Y. Bouligand, C. Salat, Tissue Cell 16 (1984) 469.

[13] M. Spencer, W. Fuller, M. H. F. Wilkins, G. L. Brown, Nature (London) 194 (1962) 1014.

[14] H. I. Petrache, N. Gouliaev, S. Tristram-Nagle, R. Zhang, R. M. Suter, J. F. Nagle, Phys. Rev. E 57 (1998) 6.

[15] C. M. Coppin, P. C. Leavis, Biophys. J. 63 (1992) 794.

[16] J. A. Spudich, R. S. Rock, Nature Cell Biology 4 (2002) 8.

[17] M. A. Geeves, Nature 415 (2002) 129.

[18] Hu, Z.; Lu, X.; Gao, J. Wang, C. Adv. Mater. 12 (2000) 1173. 
[19] Y. Okumura, K. Ito, Adv. Mater. 13 (2001) 485.

[20] T. Sakai, T, Matsunaga, Y. Yamamoto, C. Ito, R. Yoshida, S. Suzuki, N. Sasaki, M. Shibayama, U. Chung, Macromolecules 41 (2008) 5379.

[21] K. Haraguchi, T. Takeshita, Adv. Mater. 14 (2002) 1120.

[22] J. P. Gong, Y. Katsuyama, T. Kurokawa, Y. Osada, Adv. Mater. 15 (2003) 1155.

[23] J. P. Gong, Soft Matter 6 (2010) 2583.

[24] K. Naitoh, Y. Ishii, K. Tsujii, J. Phys. Chem. 95 (1991)7915.

[25] X. Chen, H. Mayama, G. Matsuo, T. Torimoto, B. Ohtani, K. Tsujii, J. of Colloid and Interf. Sci. 305 (2007) 308.

[26] J. Ozawa, G. Matsuo, N. Kamo, K. Tsujii. Macromolecules 39 (2006) 7998.

[27] K. Tsujii, M. Hayakawa, T. Onda, T. Tanaka, Macromolecules 30 (1997) 7397.

[28] M. A. Haque, G. Kamita, T. Kurokawa, K. Tsujii, J. P. Gong, Adv Mater. 22 (2010) 5110.

[29] M. A. Haque, T. Kurokawa, G. Kamita, J. P. Gong, Macromolecules 44 (2011) 8916.

[30] J.N. Israelachvili, Intermolecular \& Surface Forces, Academic Press, London, 1992, p. 227.

[31] M. A. Haque, T. Kurokawa, G. Kamita, Y. Yue, J. P. Gong, Chem. Mater. 23 (2011) 5200.

[32] W. Hong, X. Wang, Phys. Rev. E 85 (2012) 031801.

[33] J. W. Hawkes, Cell Tiss. Res. 149 (1974) 159.

[34] Y. Liu, J. Shigley, K. Hurwit, Optics Express 4 (1999) 177.

[35] K. Okumura, P. G. de Gennes, Eur. Phys. J. E. 4 (2001) 121.

[36] Y. Kang, J. J. Walish, T. Gorishnyy, E. L. Thomas, Nat. Mater. 6 (2007) 957.

[37] Z. Hu, X. Lu, J. Gao, C. Wang, Adv. Mater. 12 (2000) 1173.

[38] Y. Takeoka, M. Watanabe, Langmuir 19 (2003) 9104. 
[39] K. Matsubara, M. Watanabe, Y. Takeoka, Angew. Chem. Int. Ed. 46 (2007) 1688.

[40] Z. Hu, X. Lu, J. Gao, Adv. Mater. 13 (2001) 1708.

[41] B. Zhou, J. Gao, Z. Hu, Polymer 48 (2007) 2874.

[42] Y Iwayama, J. Yamanaka, Y. Takiguchi, M. Takasaka, K. Ito, T. Shinohara, T. Sawada, M. Yonese, Langmuir 19 (2003) 977.

[43] A. C. Arsenault, T. J. Clark, G. V. Freymann, L. Cademartiri, R. Sapienza, J. Bertolotti, E. Vekris, S. Wong, V. Kitaev, I. Manners, R. Z. Wang, S. John, D. Wiersma, G. A. Ozin, Nature Materials 5 (2006) 179.

[44] K. Sumioka, H. Kayashima, T. Tsutsui, Adv. Mater. 14 (2002) 1284.

[45] H. Fudouzi, T. Sawada, Langmuir 22 (2006) 1365. 


\section{Figure captions}

Figure 1: Iridescence phenomena of a polymerizable surfactant dodecyl glyceryl itaconate (DGI). (a) In presence of water and an ionic surfactant sodium dodecyl sulfate (SDS), DGI undergoes self-assembly and forms lamellar bilayer structure in the micro-scale exhibiting iridescent color. Molecular structure of dodecyl glyceryl itaconate (DGI) containing polymerizable double bond is also shown. (b) Iridescent color change from red to deep blue with increasing molar ratio of SDS to DGI observed at $3 \mathrm{~h}$ after mixing SDS and DGI (from left to right, the molar ratios of SDS to DGI are 1:4000, 1:2500, 1:1000, 1:600, 1:400, 1:200, $1: 100,1: 75,1: 50,1: 40$, respectively). As shown in the TEM images, when the molar ratio of SDS is 1:4000, lamellar sheets occupy the whole space but while the molar ratio of SDS is 1:40, DGI lamellae tend to curve to form onion-like and/or myelin-like structures which are separated in the water phase. Reproduced with permission from literature [25]; Copyright $\mathbb{C}$ 2007, Elsevier.

Figure 2: Formation of single domain PDGI lamellar structure. The micro-domain bilayer structure that appeared in SDS aqueous solution is retained in the mixture of acrylamide (AAm), cross-linker (MBAA), and initiator (Stage 1). The micro-domain bilayers are aligned in one direction uniformly in the macro-scale by applying shear flow to the precursor solution just before polymerization (Stage 2). By polymerization, PDGI macro-domain lamellar bilayer structures are entrapped inside the polyarylamide (PAAm) matrix and the hydrid PDGI/PAAm hydrogel is obtained (Stage 3).

Figure 3: Anisotropic PDGI/PAAm gel. (a) Photographs of PDGI/PAAm gel prepared at various concentrations of DGI $(0.11,0.13$, and $0.2 \mathrm{M})$. The lamellar bilayers are in parallel to the screen in the photographs. The scale bar in the first photographs is same for others.

The lamellar alignment is also shown in the 3D illustration of the gel. The gels exhibit magnificent structural color due to the Bragg's diffraction by the multi-layer reflection of 
visible light. The wavelength at maximum of the reflection spectrum $\left(\lambda_{\max }\right)$ was measured by fixing the Bragg's angle as $60^{\circ}$. Polarizing light microscopic images of gel prepared with high $(\sim 5 \mathrm{~cm} / \mathrm{s})$ shear flow $(\mathrm{c}, \mathrm{d})$ and no shear flow $(\mathrm{e}, \mathrm{f})$ under crossed Nicol observed from the cross-section or side as shown in ' $b$ '. The sample prepared at high shear flow produce images with contrast birefringence when rotaing by an angle of $45^{\circ}$ ( dark and bright) indicating the lamelar alignment in one direction. The gel prepared without shear flow produce images with similar birefringence level when rotaing by an angle of $45^{\circ}$ (partially bright) indicating the random lamelar orientaion. Scale bar in image 'c' is same for ' $d$ ', 'e', and ' $\mathrm{f}$ '. Reproduced with permission from literature [28]; Copyright (C) 2010 WILEY-VCH Verlag GmbH \& Co. KGaA, Weinheim.

Figure 4: One dimensional (1D) swelling behavior of the PDGI/PAAm gel. (a) Illustration of the 1D swelling behavior of gel. PAAm can swell freely in perpendicular to the lamellae in contrast parallel to lamellae where the free swelling of PAAm is completely constrained by the non-swellable lamellar layers. (b) Swelling ratio of the PDGI/PAAm gel increases with time along the thickness axis (perpendicular to the bilayers; $\sim 2.2$ times), while no swelling at all along the length axis (parallel to the bilayers). Reproduced with permission from literature [31]; Copyright (C) 2011, American Chemical Society.

Figure 5: Stratified layer structure model of PDGI/PAAm gel. (a) Illustration of the swelling-nonswelling layers (SL-NL) structure of a lamellar gel. The as-synthesized state is taken to be the reference, in which the SL already contains solvent. The overall stretches are $\lambda_{1}, \lambda_{2}$, and $\lambda_{3}$, while the through-thickness stretch of the SLs is $\lambda_{3}$. (b) Equilibrium stretches of a free-swelling PDGI/PAAm lamellar gel, parallel $\left(\lambda_{1}\right)$ and perpendicular $\left(\lambda_{3}\right)$ to the lamellae, as functions of the DGI monomer concentration. (c) Tensile moduli of the PDGI/PAAm gel parallel $\left(E_{1}\right)$ and perpendicular $\left(E_{3}\right)$ to the lamellae as functions of the DGI concentration. 
Squares and circles are experimental results from Ref. [28]. Reproduced with permission from literature [32]; Copyright (C2012 American Physical Society.

Figure 6: Reversible color tuning of the PDGI/PAAm gel by compressive deformation. (a) The gel exhibits a color shift from red to blue upon gradual increase in compressive strain (right to left) perpendicular to the lamellar layer, and corresponding reflection spectra showing the peak position shift from right to left. The reflection spectrum was measured by fixing the Bragg's angle as $60^{\circ}$. (b) The wavelength maximum, $\lambda_{\max }$, and lamellar distance, $d$, decrease linearly with increasing strain up to a strain of around 0.45 . The compressive strain is mainly localized in the soft PAAm network layers of the gel, which causes a decrease in lamellar distance in the perpendicular direction resulting in the blue shift of color. The diameter of the disk shape gel sample is $10 \mathrm{~mm}$ at zero strain. Reproduced with permission from literature [28]; Copyright (C) 2010 WILEY-VCH Verlag GmbH \& Co. KGaA, Weinheim.

Figure 7: Reversible color tuning of the PDGI/PAAm gel by tensile elongation. (a) The PDGI/PAAm gel changes its color from red to blue upon gradual increase in tensile strain (left to right) applied parallel to the lamellar layer. (b) The wavelength maximum, $\lambda_{\max }$, decreases with increasing strain. The tensile strain is mainly caused a decrease in the gel thickness resulting in a decrease in lamellar distance, $d$, in the perpendicular direction along with the corresponding blue shift of color. The reflection spectrum was measured by fixing the Bragg's angle as $60^{\circ}$. The scale bar is $5 \mathrm{~mm}$ for the gel sample at zero strain. Reproduced with permission from literature [29]; Copyright (C) 2011, American Chemical Society. 
(a)<smiles>C=C(CC(=O)OCCCCCCCCCCCC)C(=O)OCC(O)CO</smiles>

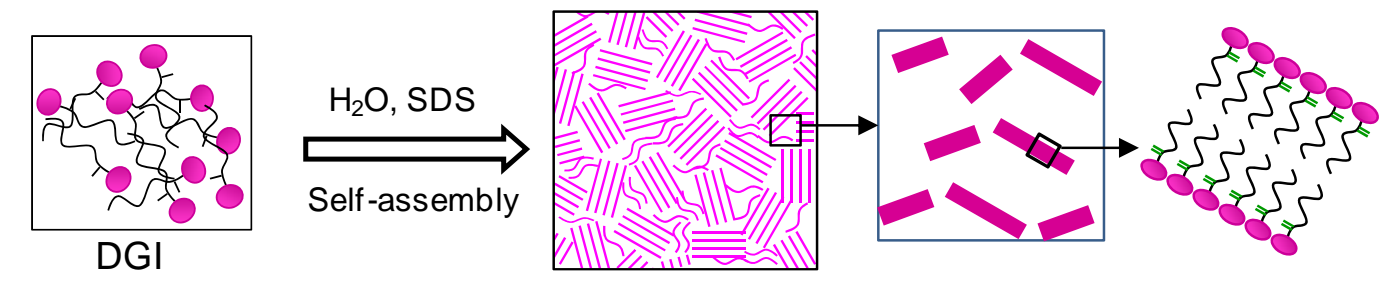

(b)

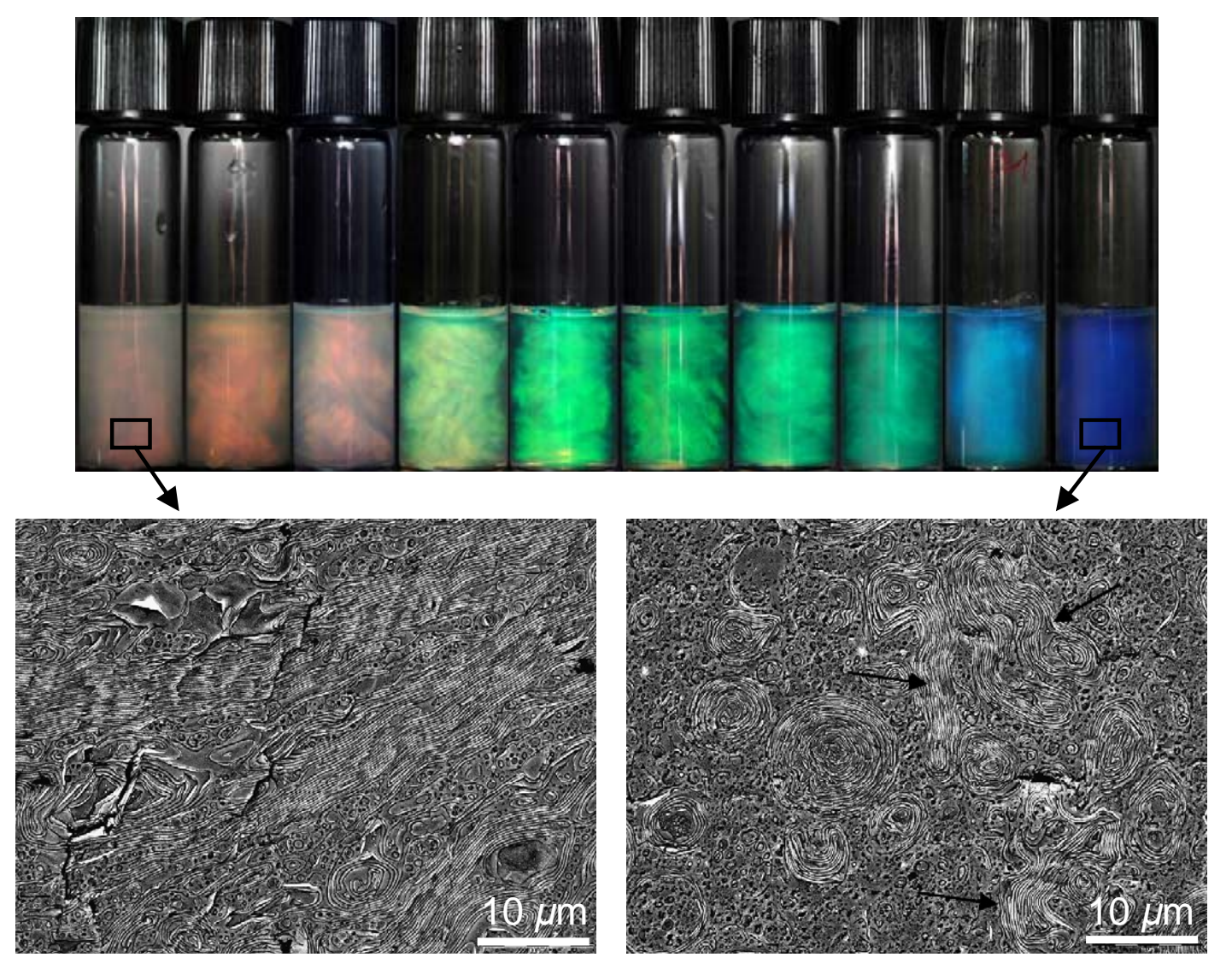

Figure 1 
(1)

(2)

(3)

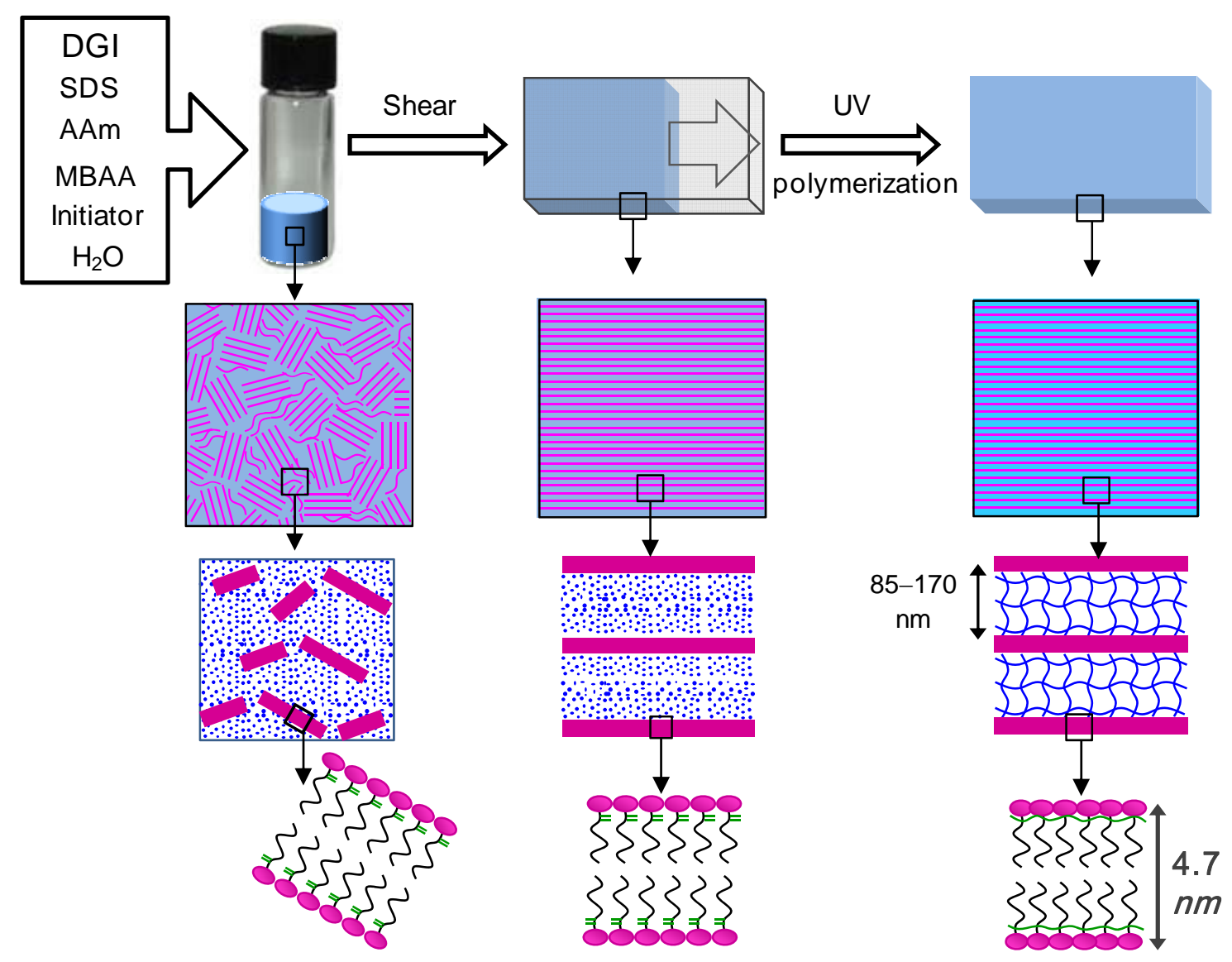

Figure 2 

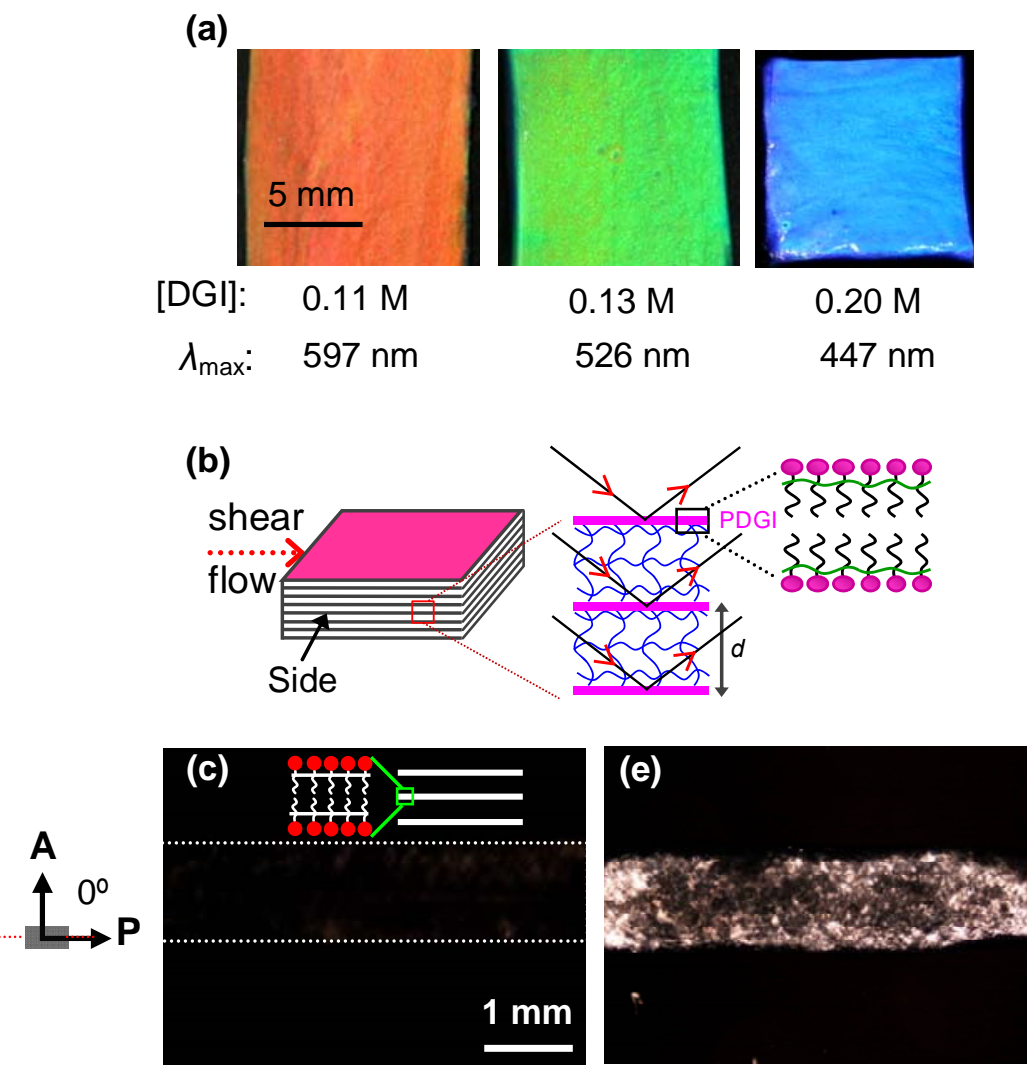

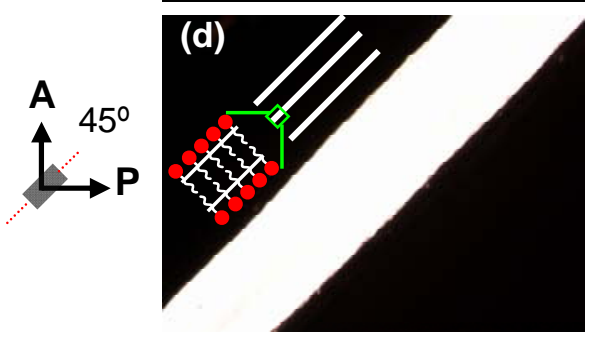

Strong shear flow

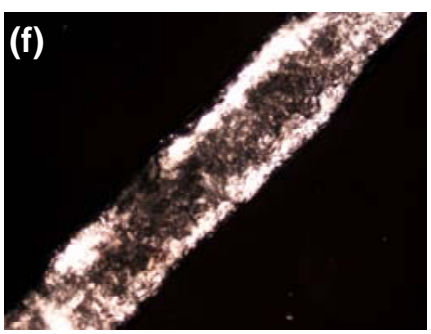

No shear flow

Figure 3 
a)

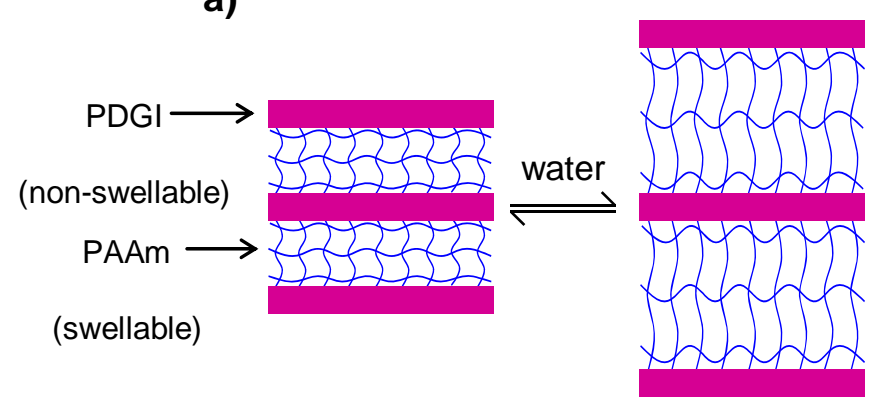

b)

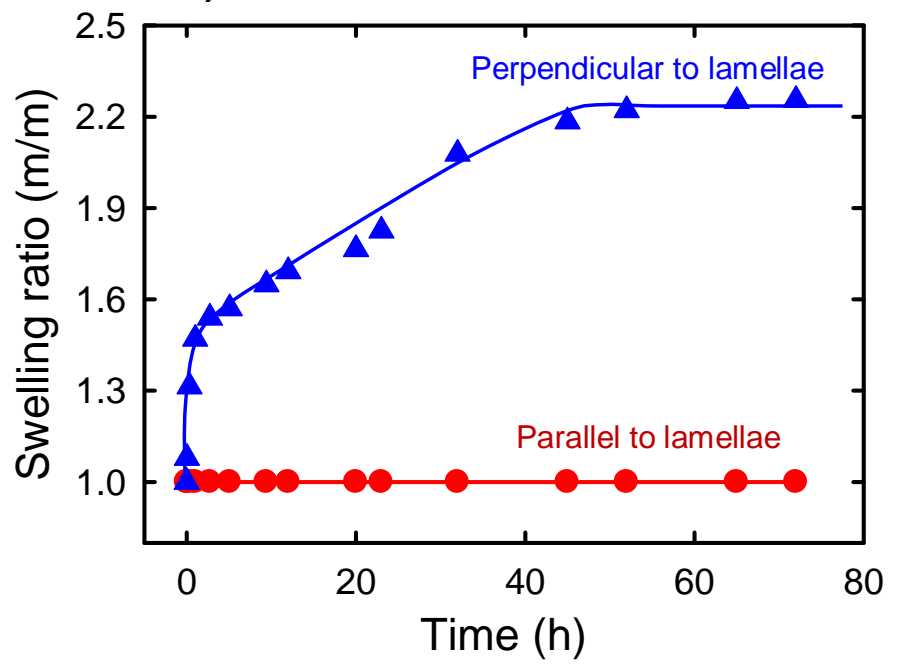

Figure 4 
(a)
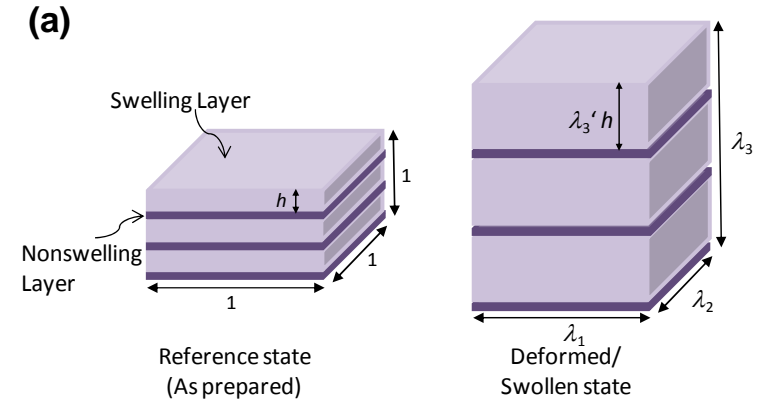

(b)

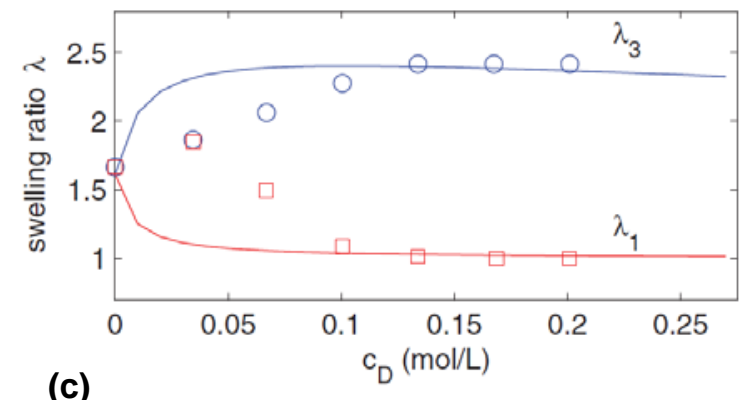

(c)

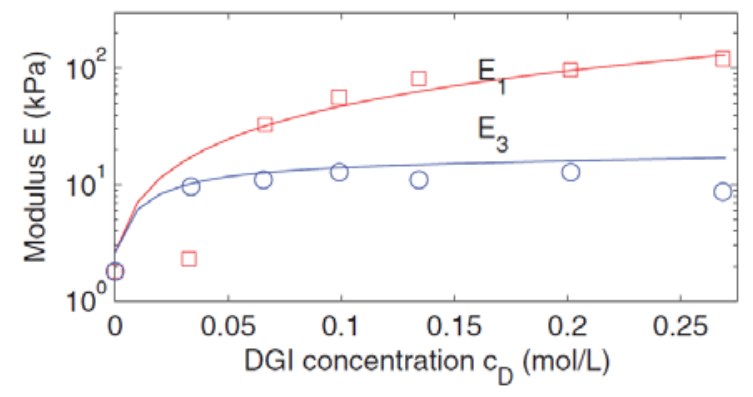

Figure 5 
(a)

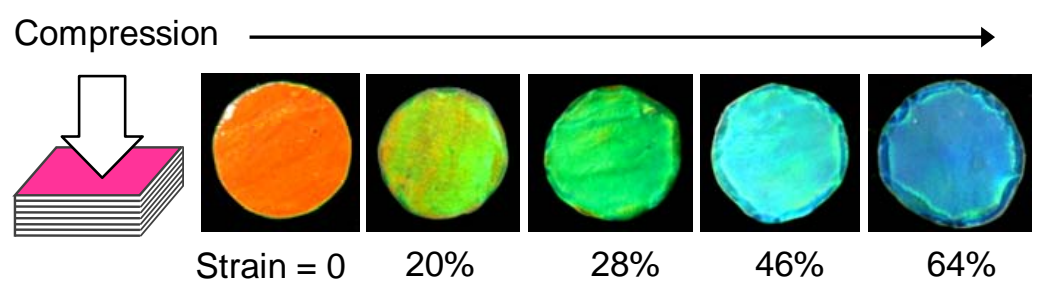

(b)

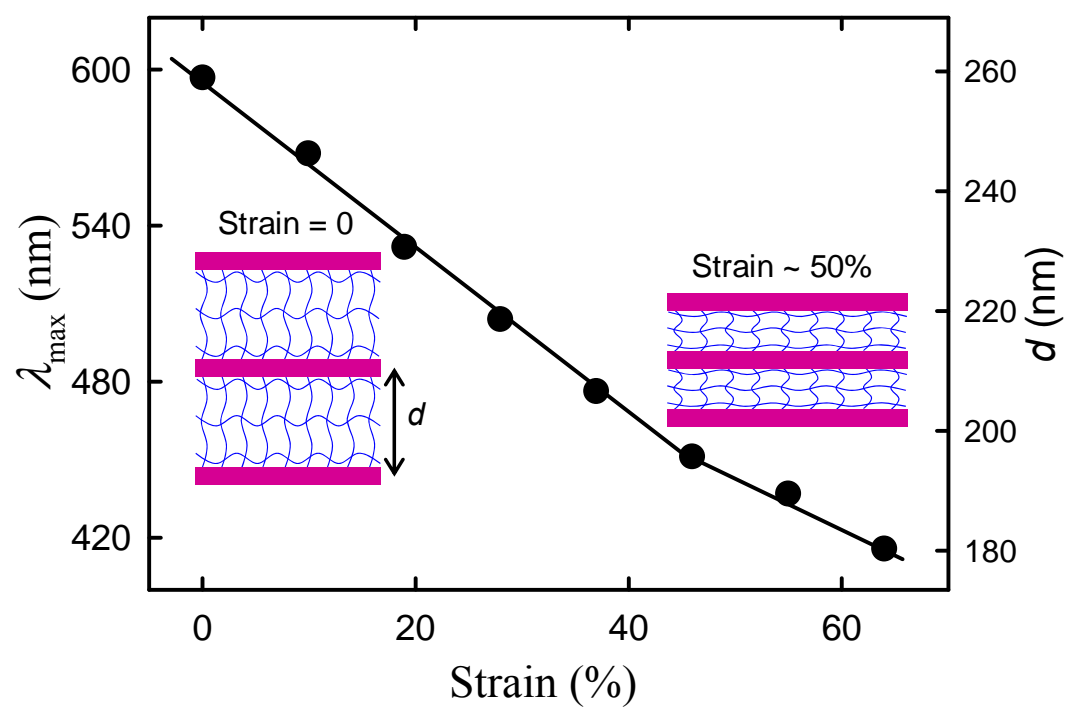

Figure 6 
(a)

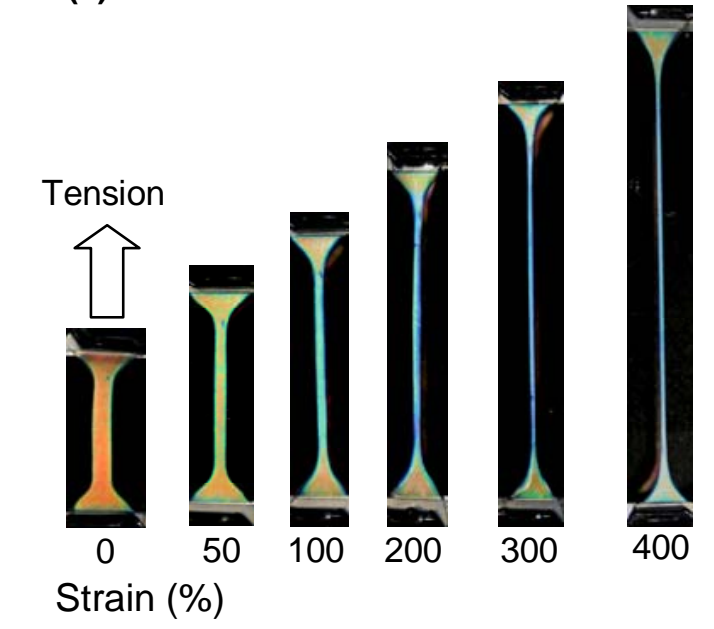

(b)

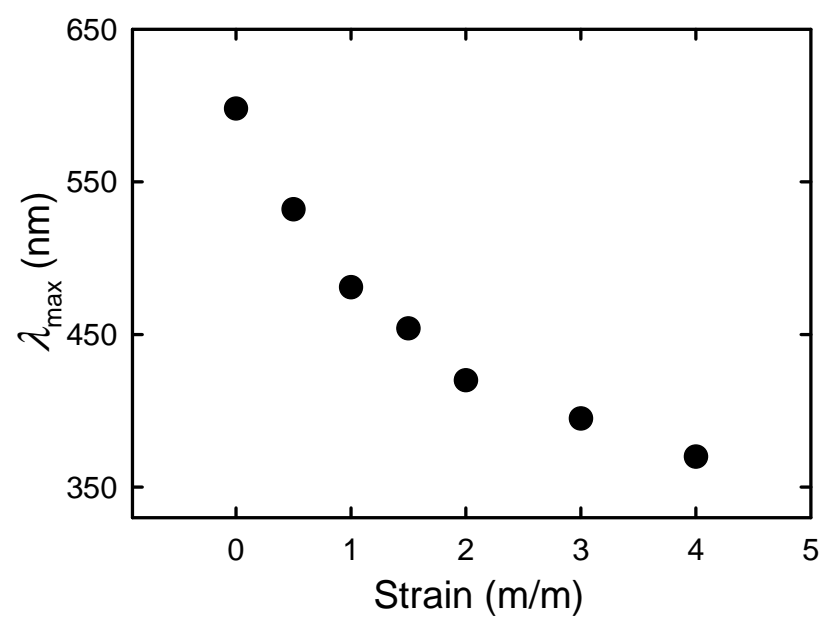

Figure 7 